\title{
TITULACIÓN POR TESIS EN ESCUELAS DE MEDICINA DE LIMA, 2011: CARACTERÍSTICAS, MOTIVACIONES Y PERCEPCIONES
}

\author{
Christian R. Mejia ${ }^{1,2, a}$, Fiorella Inga-Berrospij,a, Percy Mayta-Tristán ${ }^{1, a}$
}

\begin{abstract}
RESUMEN
Se encuestó a los médicos titulados por tesis de las siete escuelas de Medicina de Lima en el 2011 de las siete escuelas de Medicina de Lima para conocer las características del proceso de titulación por tesis, sus motivaciones y sus percepciones sobre dicho proceso. Se incluyó 98 tesistas ( $87 \%$ del total), $99 \%$ realizó tesis observacionales y el $35 \%$ realizó la tesis de manera individual (solo un tesista) en grupo de tres. La principal motivación fue que era bueno para su currículo (94\%). En aquella universidad donde la tesis es obligatoria se empezó antes la elección del tema y el asesor. La percepción de mayor y menor dificultad en el proceso fue la realización de los trámites administrativos (53\%) y seleccionar a su asesor (11\%), respectivamente. Se deben revisar los procesos y tiempos administrativos para que no dificulten la realización de tesis, puesto que la nueva Ley Universitaria obliga a la elaboración de tesis para titularse.
\end{abstract}

Palabras clave: Tesis; Educación médica; Investigación; Estudiantes de medicina; Perú (fuente DeCS BIREME).

\section{MEDICAL DEGREE EARNED WITH A THESIS IN MEDICAL SCHOOLS OF LIMA, 2011: CHARACTERISTICS, MOTIVATIONS AND PERCEPTIONS}

\begin{abstract}
We surveyed physicians who obtained their medical degree with a thesis in 2011 from the seven medical schools in Lima to know the characteristics of the degree by thesis process, as well as participants' motivations and perceptions of that process. We included 98 students who did a thesis ( $87 \%$ of total); $99 \%$ conducted observational thesis, $30 \%$ did so in groups of three. The main motivation was that it was good for their curriculum vitae (94\%). At the university where the thesis is compulsory, the process began with the choice of topic and adviser. Perceived "greatest" and "least" difficulty in the process was the completion of administrative procedures $(53 \%)$ and selection of their advisor (11\%), respectively. Administrative timeliness and processes should be reviewed so as not to impede the completion of thesis, since the new University Act requires the completion of a thesis to graduate.
\end{abstract}

Key words: Academic dissertations; Education, medical; Research; Students, medical; Peru (source: MeSH, NLM).

\section{INTRODUCCIÓN}

La obtención del título profesional es la culminación del período de formación universitaria y requisito indispensable para ejercer la medicina en el Perú. Hasta 1991 la única vía de titulación era la aprobación y sustentación de la tesis ${ }^{(1)}$, luego se implementaron otras formas de graduación en algunas universidades (2). En los últimos años, la cantidad de estudiantes que se titulan por tesis sigue siendo baja, a pesar que los estudiantes de Medicina manifiestan que tienen la intención de hacer una tesis ${ }^{(3)}$ y que la tesis representa uno de los fines principales de la universidad -la investigación- además, esto sirve como indicador de producción científica ${ }^{(4,5)}$.

Adicionalmente, muchos estudiantes sienten que no cuentan con las herramientas necesarias para investigar y publicar desde el pregrado ${ }^{(6,7)}$, herramientas que son básicas para llevar adecuadamente una investigación. Las dificultades mencionadas con mayor frecuencia son el no contar con una adecuada asesoría, el déficit en el conocimiento metodológico, el apoyo en los procesos administrativos, entre otros ${ }^{(5,8)}$. Esta realidad

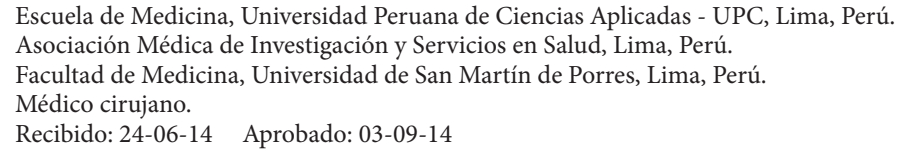


genera que el examen de grado y/o curso de suficiencia sean actualmente las modalidades más usadas para acceder a la titulación de médicos ${ }^{(3,9)}$. La mayoría de estudios en el Perú abordan los temas de capacitación, investigación y publicación, pero casi no se tienen reportes de la frecuencia de tesis en el pregrado, así como de las motivaciones, dificultades y percepciones de los tesistas ${ }^{(10,11)}$.

El objetivo del estudio fue determinar las características del desarrollo y sustentación de la tesis, así como las motivaciones y percepciones en médicos que se titularon por tesis en escuelas de Medicina de Lima en el 2011.

\section{EL ESTUDIO}

Se realizó un estudio de corte transversal entre los meses abril y mayo de 2011, aprobado por el Comité de Ética de la Universidad de San Martín de Porres (1585 CIEUSMP-CCM). Se incluyó a todos los médicos colegiados hasta abril de 2011 en el Consejo Regional III del Colegio Médico del Perú, quienes realizaron el internado en el 2010 y se titularon por tesis en alguna de las escuelas de Medicina ubicadas en Lima Metropolitana, una donde la tesis es obligatoria (Universidad Peruana Cayetano Heredia -UPCH) y seis donde es opcional (Universidad Científica del Sur, Universidad Nacional Federico Villarreal, Universidad Nacional Mayor de San Marcos, Universidad Ricardo Palma, Universidad Privada San Juan Bautista y Universidad de San Martín de Porres), se excluyó a los que no deseaban participar o no hayan podido ser localizados.

Al no haber listas oficiales o un prerregistro de los tesistas por cada universidad, se realizó un muestreo por bola de nieve; primero, se ubicó a los delegados de cada universidad quienes ayudaron a ubicar a sus colegas que sustentaron tesis, luego ellos refirieron a otros, hasta tener a la mayor cantidad de tesistas hasta abril de 2011. En el 2013 se corroboró la cantidad final de tesistas cruzando información con las tesis disponibles en las bibliotecas de cada facultad (Tabla 1).

Se elaboró una encuesta autoadministrada, que incluyó las características generales de los participantes, antecedentes académicos y de investigación, descripción del desarrollo y sustentación de la tesis, así como las motivaciones y percepciones sobre las dificultades que tuvieron durante el proceso de graduación por tesis.

Para la construcción del instrumento y la forma de contactar a los participantes se consideró investigaciones similares ${ }^{(3,11,12)}$ y se consultó, por lo menos, a un titulado en años previos por la modalidad de tesis en las universidades incluidas. En los casos donde no hubo
Tabla 1. Cantidad, de tesistas incluidos y encuestados de las facultades de Medicina de Lima, Perú, 2011

\begin{tabular}{|c|c|c|c|}
\hline Universidad & $\begin{array}{c}\text { Tesistas hasta } \\
\text { abril del } 2011\end{array}$ & $\begin{array}{c}\text { Contactados } \\
\mathrm{n}(\%)\end{array}$ & $\begin{array}{c}\text { Encuestados } \\
\mathrm{n}(\%)\end{array}$ \\
\hline $\begin{array}{l}\text { U. Peruana } \\
\text { Cayetano Heredia* }\end{array}$ & 79 & $70(88,6)$ & $70(100)$ \\
\hline $\begin{array}{l}\text { U. de San Martín } \\
\text { de Porres }\end{array}$ & 24 & $19(79,1)$ & $13(68,4)$ \\
\hline $\begin{array}{l}\text { U. Nacional Mayor } \\
\text { de San Marcos }\end{array}$ & 10 & $9(90)$ & $9(100)$ \\
\hline U. Ricardo Palma & 5 & $5(100)$ & $5(100)$ \\
\hline U. Científica del Sur & 1 & $1(100)$ & $1(100)$ \\
\hline $\begin{array}{l}\text { U. Nacional Federico } \\
\text { Villarreal }\end{array}$ & 0 & -- & -- \\
\hline $\begin{array}{l}\text { U. Privada San Juan } \\
\text { Bautista }\end{array}$ & 0 & --- & --- \\
\hline Total & 119 & $104(87,4)$ & $98(94,2)$ \\
\hline
\end{tabular}

$\mathrm{U}$ : universidad, * universidad con modalidad de titulación por tesis obligatoria

graduados, se consultó a miembros de las sociedades científicas estudiantiles para conocer los procesos administrativos implicados en la titulación por tesis. Luego, fue revisado por tres docentes de investigación de universidades peruanas, por último, se hizo un piloto con diez médicos que se titularon por tesis en el mismo periodo, pero de universidades del interior del país.

Las seis preguntas que medían las motivaciones del por qué decidieron realizar su tesis fueron medidas en escala likert de cinco opciones (que iban desde muy de acuerdo a muy en desacuerdo); para la presentación gráfica de resultados se juntó las categorías de acuerdo y muy de acuerdo (categoría de interés).

Se evaluó la percepción sobre el nivel de dificultad que tuvo el tesista para realizar cada una de las fases de la tesis (selección de idea de investigación, del asesor, elaboración de protocolo, aprobación de protocolo, ejecución del estudio, análisis de datos, elaboración de informe final, aprobación de informe final, trámites administrativos y sustentación), usando una escala de likert de cinco opciones (desde muy fácil a muy difícil).

Así mismo, se indagó sobre los procesos de revisión y aprobación del comité de ética en investigación, de los asesores y las modificaciones que recibieron en el proceso; finalmente, se describió las características del estudio y los procedimientos utilizados para la recolección de datos.

Se contactó a cada persona vía telefónica para corroborar que haya realizado tesis para titularse e invitándole a participar del estudio, al cual daba su consentimiento verbal. A quienes aceptaron participar 
se les envió por correo electrónico el link del acceso para contestar la encuesta, que estaba disponible en el programa SurveyMonkey®, de no obtener respuesta en una primera oportunidad se contactó por correo electrónico o teléfono hasta en dos oportunidades más, en el lapso de una semana. De no obtener respuesta se le consideró como rechazo.

Los datos fueron exportados de la plataforma virtual del Survey Monkey ${ }^{\circledR}$ al programa Excel® 2007 y fueron procesados en el paquete estadístico STATA $®$ 11.0. Se realizó un análisis descriptivo usando frecuencias y porcentajes para las variables categóricas, y se evaluó las variables numéricas con la prueba estadística Shapiro Wilk, al determinar que no tenían comportamiento normal se las describió con medianas y rangos. Se realizaron comparaciones según la obligatoriedad de la tesis en su universidad usando las pruebas $\mathrm{Chi}^{2}$ de Pearson, la exacta de Fisher y la suma de rangos de Wilcoxon, según correspondía. Se consideró un valor $p<0,05$ como significativo.

\section{HALLAZGOS}

De los 119 médicos que se titularon por tesis en alguna universidad de Lima entre enero y abril del 2011, el $87,4 \%$ (104) pudo ser contactado, de los cuales, el $94,2 \%$ (98) participó en el estudio, la tasa de rechazo fue de $5,8 \%$ (Tabla 1 ).

\section{CARACTERÍSTICAS DE LOS TESISTAS Y EL PROCESO DE TITULACIÓN}

De los 98 médicos que participaron en el estudio, el $60 \%$ (59/98) fue de sexo femenino, la mediana de edad fue de 25 años (rango 22-37 años); 71\% (70/98) provinieron de una universidad donde la tesis es obligatoria y el $90,8 \%$ (89/98) egresó de alguna universidad privada (Tabla 2).

De los antecedentes de investigación en el pregrado, el $31,6 \%(31 / 98)$ de los encuestados manifestó haber participado en un curso extracurricular en investigación, la misma cantidad formó parte de su sociedad científica estudiantil local; el 50\% (49/98) refirió haber realizado una investigación extracurricular; el 29,6\% (29/98) presentó un trabajo a un congreso científico y el 38,8\% (38/98) publicó en una revista científica durante el pregrado.

El $35,1 \%$ (34/97) manifestó que desarrolló su tesis de manera individual; el $34,0 \%$ (33/97) en pares y el 30,9\% (30/97) en grupo de a tres. El 99,0\% de los diseños de investigación fue de tipo observacional, siendo los más frecuentes los estudios transversales $(36,7 \%)$ y series de casos $(26,5 \%)$.
Tabla 2. Características de los tesistas y el proceso que siguieron según obligatoriedad de titularse por tesis en las facultades de Medicina de Lima, 2011

\begin{tabular}{|c|c|c|c|}
\hline \multirow[t]{2}{*}{ Variables } & \multicolumn{2}{|c|}{$\begin{array}{l}\text { Obligatoriedad } \\
\text { de la tesis N (\%) }\end{array}$} & \multirow{2}{*}{ Valor $p$} \\
\hline & Obligatoria & Opcional & \\
\hline $\begin{array}{l}\text { Edad } \\
\text { (mediana y rango) }\end{array}$ & $27(23-37)$ & $24,5(22-30)$ & $<0,001^{*}$ \\
\hline \multicolumn{4}{|l|}{ Sexo } \\
\hline Masculino & $28(40,0)$ & $11(39,3)$ & $0,948^{* *}$ \\
\hline Femenino & $42(60,0)$ & $17(60,7)$ & \\
\hline \multicolumn{4}{|l|}{ Estado civil } \\
\hline Soltero & $69(98,6)$ & $26(92,9)$ & $0,196 \$$ \\
\hline Conviviente & $1(1,4)$ & $2(7,1)$ & \\
\hline \multicolumn{4}{|l|}{ Lugar de nacimiento } \\
\hline Lima-Callao & $50(71,4)$ & $12(42,9)$ & $0,008^{* *}$ \\
\hline Provincias & $20(28,6)$ & $16(57,1)$ & \\
\hline \multicolumn{4}{|c|}{ Tiempo en que terminó la carrera } \\
\hline En 7 años & $58(85,3)$ & $13(46,4)$ & $<0,001 \$$ \\
\hline Un año más & $9(13,2)$ & $5(17,9)$ & \\
\hline Dos o más años & $1(1,5)$ & $10(35,7)$ & \\
\hline \multicolumn{4}{|c|}{ Dominio del idioma inglés } \\
\hline Básico & $1(1,4)$ & $7(25,0)$ & $<0,001^{* *}$ \\
\hline Intermedio & $19(27,2)$ & $16(57,1)$ & \\
\hline Avanzado & $50(71,4)$ & $5(17,9)$ & \\
\hline \multicolumn{4}{|l|}{ Tiene familiares médicos } \\
\hline Sí & $46(65,7)$ & $23(82,1)$ & $0,107^{\star *}$ \\
\hline No & $24(34,3)$ & $5(17,9)$ & \\
\hline \multicolumn{4}{|c|}{ En 5 años donde planea estar laborando } \\
\hline Extranjero & $50(71,4)$ & $12(42,9)$ & $0,019 \$$ \\
\hline Lima & $18(25,7)$ & $15(53,5)$ & \\
\hline Provincia & $2(2,9)$ & $1(3,6)$ & \\
\hline \multicolumn{4}{|c|}{ La aprobación del proyecto de tesis fue... } \\
\hline Antes del internado & $23(34,3)$ & $1(3,6)$ & $<0,001^{* *}$ \\
\hline Durante el internado & $18(26,9)$ & $22(78,6)$ & \\
\hline Después del internado & $26(38,8)$ & $5(17,8)$ & \\
\hline
\end{tabular}

*Prueba Suma de rangos de Wilcoxon. ** Prueba chi cuadrado. \$ Prueba exacta de Fisher

El 14,3\% (14/98) de los encuestados refirió que sus proyectos fueron aprobados por un comité de ética y el $6,1 \%$ (6/98) logró obtener un financiamiento para desarrollar la investigación; de ellos, a tres los financió su universidad y los otros tres obtuvieron recursos de otra institución.

\section{MOTIVACIONES DE LOS TESISTAS}

El 93,9\% (92/98) contestó que una de sus motivaciones para realizar la tesis es porque saben que es bueno para su curriculum vitae; el 82,7\% (81/98) manifestó que una de sus motivaciones era que les gusta investigar, y el $49,0 \%$ (44/98) refirió que lo hicieron porque se quieren dedicar a la investigación en el futuro.

Cuando se evaluó las motivaciones para realizar la tesis según la obligatoriedad en su universidad, se encontró diferencia significativa según si manifestó que 


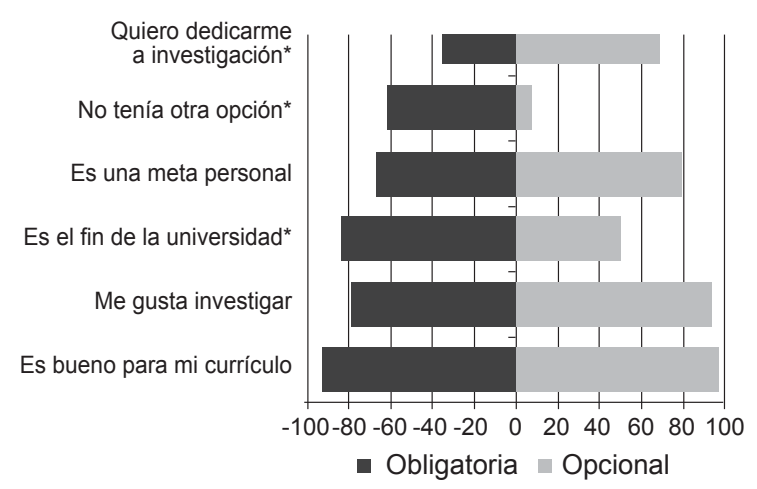

Figura 1. Motivación para la realización de tesis según obligatoriedad en egresados de las facultades de Medicina de Lima, 2011 ${ }^{*} p<0,05$ (prueba de suma de rangos de Wilcoxon).

su motivación fue el que deseaba ser investigador en el futuro $(p=0,004)$, que no tenían otra opción para titularse $(p<0,001)$ y aquel que pensó que la tesis era el fin de la universidad $(p=0,001)$; (Figura 1).

\section{PERCEPCIONES SOBRE EL PROCESO DE TITULARSE MEDIANTE TESIS}

El proceso que generó mayores dificultades fue el de los trámites administrativos $(52,7 \%)$, junto con la elaboración del informe $(51,1 \%)$ y la aprobación del protocolo $(43 \%)$. El seleccionar a su asesor ( $11 \%$ ) fue percibido como la menor dificultad en el proceso, los alumnos de la universidad donde la tesis fue obligatoria percibió la aprobación del informe como más fácil $(p=0,008)$ (Figura 2).

\section{DISCUSIÓN}

Estos resultados son un insumo importante en el escenario universitario actual, donde se acaba de aprobar una nueva Ley Universitaria que restablece

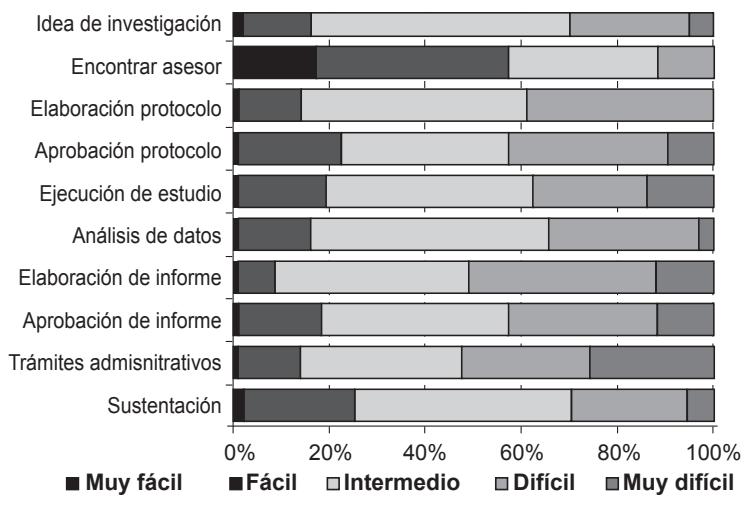

Figura 2. Percepciones sobre la dificultad de los procesos relacionados con la graduación por tesis en egresados de las facultades de Medicina de Lima, 2011 la obligatoriedad de la tesis para obtener el título en el Perú ${ }^{(13)}$, particularmente, considerando que la percepción de la mayor dificultad en el proceso de tesis fue la realización de los trámites administrativos. Esto implica que los procesos para la realización de tesis en las escuelas de medicina desaniman su ejecución en universidades donde no era obligatoria (11) y ahora deben ajustar sus procesos para que sean más fluidos y puedan soportar una carga mayor de tesistas.

La percepción de lo más sencillo fue el encontrar a un asesor de tesis, aspecto que no guarda relación con la bibliografía encontrada, donde son comunes las dificultades para encontrar a un asesor adecuado $(5,8,14,15)$. Esto podría explicarse porque se interrogó acerca de la facilidad para encontrar a un asesor de tesis, pero no se evaluó el desempeño y conocimiento del tema por parte de estos, el tiempo a disposición, los aportes e, incluso, la motivación que le dio para realizar su tesis. En la UPCH, donde la tesis es obligatoria, hay mayor disponibilidad de asesores por el número de investigadores que albergan; en las otras universidades, a pesar de ser la tesis un evento poco frecuente, tampoco es un problema el encontrar un asesor; sin embargo, será un reto tener el número adecuado de asesores para todo el volumen de alumnos nuevos que harán tesis en las universidades donde no era obligatoria.

Pocos tesistas refirieron haber participado en un curso extracurricular de investigación o haber pertenecido a su sociedad científica local, cifras que coinciden con investigaciones nacionales, que refieren que son pocos los interesados en acercarse de esta manera a la investigación ${ }^{(6,11)}$, peroque son menoresalas encontradas en Brasil, donde más de la mitad de estudiantes del pregrado realizó algún curso extracurricular relacionado con la investigación ${ }^{(14)}$. La cantidad de tesistas que participaron en alguna investigación extracurricular fue similar a lo encontrada en múltiples estudios, lo que nos sugiere que los interesados en la investigación desde el pregrado se involucran en este tipo de actividades por iniciativa propia ${ }^{(6,14,16)}$. Muy pocos presentaron su trabajo en algún congreso científico o publicaron en alguna revista indizada durante el pregrado, a comparación de los encontrado en bibliografía extranjera, donde el porcentaje es mayor en ambos campos, esto muestra que, a pesar de tener porcentajes similares de participación en investigación, muchos no logran finalizar el proceso y presentar los resultados a la comunidad científica ${ }^{(16,17)}$.

Según el diseño de las tesis, casi la totalidad fueron de tipo no experimental, y el tipo descriptivo fue el más común, esto guarda relación con lo encontrado en estudios bibliométricos de las tesis en dos universidades 
estatales peruanas y una particular ${ }^{(4,9,18)}$. Sin embargo, en otros países, la mayoría de tesis son de carácter experimental ${ }^{(19)}$, esta diferencia puede estar relacionada al acceso a fondos de investigación y laboratorios bien implementados.

Las principales motivaciones encontradas fueron el saber que titularse por tesis era bueno para el curriculum vitae y hacerlo por el gusto a investigar. Esto guarda relación con lo encontrado por algunos investigadores nacionales (11,21), donde prima el saber que lo que harán será parte importante de su hoja de vida y que lo realizan por un interés personal a la investigación. Otra motivación importante, que tampoco tuvo asociación con la obligatoriedad de realizar tesis, fue el que el investigar sea una meta personal del estudiante, que concuerda con otras investigaciones a nivel nacional e internacional (15,21). El favorecer el pensamiento de los estudiantes de que la tesis es útil para conseguir futuros logros profesionales, en el campo académico, así como el reforzar su percepción de objetivo personal. Estas serían las motivaciones más importantes a ser evaluadas por los formadores, autoridades e instituciones interesadas en mejorar la investigación desde el pregrado ${ }^{(22)}$.

Una limitación del estudio es la selección de los sujetos de estudio, ya que no se pudo obtener el listado oficial de tesistas de cada universidad; sin embargo, se incluyó a 98 de 119 (82,4\%). Por otro lado, no fue posible incluir a más tesistas, pues no habían, al punto que en dos universidades ningún titulado optó por esa modalidad. Si bien con tan pocos casos en algunas universidades es difícil caracterizar el proceso de titulación por tesis, no es posible hacerlo de otra manera si es que no hay más estudiantes de Medicina que opten por titularse por tesis.

En conclusión, la mayoría de tesis sustentadas en el 2011 en las escuelas de Medicina son estudios observacionales, que son elaborados y ejecutados hasta el último año de Medicina, las motivaciones más importantes son el saber que será bueno para su currículo así como el gusto por investigar y que la percepción de la mayor dificultad en el proceso de tesis fue en los trámites administrativos.

Contribuciones de autoría: CRM, FIB y PMT tuvieron la idea de investigación y diseñaron el estudio, CRM y FIB recopilaron los datos, PMT y CRM realizaron el análisis e interpretación de datos, todos los autores participaron en la redacción del manuscrito y aprobaron la versión final a publicar.

Conflicto de interés: los autores declaran no tener conflictos de interés con la publicación de este artículo.

Fuentes de financiamiento: autofinanciado.

\section{REFERENCIAS BIBLIOGRÁFICAS}

1. Perú, Ministerio de Educación. Decreto Legislativo 739: Establecen normas mínimas de exigencia académica a fin de facilitar a los estudiantes universitarios la obtención de sus grados académicos que les permita acceder a puestos de trabajo. Lima: Congreso de la República; 1991.

2. Colegio Médico del Perú. Cuadernos de Debate en Salud Pública - Calidad de la educación en la formación médica. En: Zavala Sarrio SW. ¿Con qué perfil y competencias debe egresar el médico en los próximos años en el Perú? Lima: Colegio Médico del Perú; 2010. p. 32-5.

3. Galán-Rodas E, Díaz-Vélez C, Mejia CR. Intención en la modalidad de titulación de internos de Medicina en el Perú, 2010. Rev Peru Med Exp Salud Publica. 2011;28(2):390-1.

4. Valle R, Salvador E. Análisis bibliométrico de las tesis de pregrado de la Facultad de Medicina de la Universidad Nacional Mayor de San Marcos. An Fac Med. 2009;70(1):11-8.
5. San Martín HF, García PM. La tesis y su problemática en la Facultad de Medicina Veterinaria de la UNMSM. Rev Inv Vet Peru. 2006;17(1):81-8.

6. Díaz Velez C, Manrique González LM, Galán Rodas E, Apolaya Segura M. Conocimientos, actitudes y prácticas en investigación de los estudiantes de pregrado de facultades de medicina del Perú. Acta Med Per. 2008 EneMar;25(1):9-15.

7. Valle R, Salvador E. La tesis de medicina humana: Experiencia en una universidad pública de Lima. Rev Peru Med Exp Salud Publica. 2009 AbrJun;26(2):268-70.

8. Arroyo-Hernández $\mathrm{CH}$, De la Cruz W, Miranda-Soberon UE. Dificultades para el desarrollo de investigaciones en pregrado en una universidad pública de provincia, Perú. Rev Peru Med Exp Salud Publica. 2008 OctDic;25(4):448-9.

9. Taype-Rondán A, Carbajal-Castro C, Arrunategui-Salas G, Chambi-Torres
J. Limitada publicación de tesis de pregrado en una facultad de medicina de Lima, Perú, 2000-2009. An Fac Med. 2012;73(2):153-7.

10. Arriola-Quiroz I, Curioso WH, Cruz-Encarnacion M, Gayoso O. Characteristics and publication patterns of theses from a Peruvian medical school. Health Info Libr J. 2010 Jun;27(2):148-54. doi: 10.1111/j.1471-1842.2010.00878.x.

11. Ramos-Rodríguez MI, Sotomayor R. Realizar o no una tesis: razones de estudiantes de medicina de una universidad pública y factores asociados. Rev Peru Med Exp Salud Publica. 2008 Jul-Set;25(3):322-4.

12. Darien M, Wallet F, Schoeffler M, Tassin C, Lieutaud T, Piriou V. [Profile and motivations of students performing a universitary degree in 2011]. Ann Fr Anesth Reanim. 2012 Nov;31(11):840-5. doi: 10.1016/j. annfar.2012.05.009. [Article in French]. 
13. Perú, Congreso de la República. Ley 30220: Ley Universitaria. Lima: Congreso de la República; 2014.

14. de Oliveira NA, Luz MR, Saraiva RM, Alves LA. Student views of research training programmes in medical schools. Med Educ. 2011 Jul;45(7):748-55. doi: 10.1111/j.1365-2923.2011.03986.x.

15. Viola FJ. Elaboración de tesis: la crisis necesaria. Revista Humanidades Medicas. 2010;10(2):1-9.

16. Hunskaar S, Breivik J, Siebke M, Tømmerås K, Figenschau K, Hansen JB. Evaluation of the medical student research programme in Norwegian medical schools. A survey of students and supervisors. BMC Med Educ. 2009 Jul 14;9:43. doi: 10.1186/1472-6920-9-43.

17. Dyrbye LN, Davidson LW, Cook DA. Publications and presentations resulting from required research by students at Mayo Medical School, 1976-2003. Acad Med. 2008 Jun;83(6):604-10. doi: 10.1097/ ACM.0b013e3181723108.

18. Saldaña-Gastulo JJC, Quezada-Osoria CC, Peña-Oscuvilca A, Mayta-Tristán P. Alta frecuencia de plagio en tesis de medicina de una universidad pública peruana. Rev Peru Med Exp Salud Publica. 2010;27(1):63-7.

19. Nieminen P, Sipilä K, Takkinen HM, Renko M, Risteli L. Medical theses as part of the scientific training in basic medical and dental education: experiences from Finland. BMC Med Educ. 2007 Dec 5;7:51.

20. Gutiérrez C, Mayta P. Publicación desde el pregrado en latinoamérica: importancia, limitaciones y alternativas de solución. CIMEL. 2003;8(1):54-60.
21. Ogunyemi D, Bazargan M, Norris K, Jones-Quaidoo S, Wolf K, Edelstein R, et al. The development of a mandatory medical thesis in an urban medical school. Teach Learn Med. 2005 Fall;17(4):363-9.

22. Ruiz Dodobara F. Relación entre la motivación de logro académico, la autoeficacia y la disposición para la realización de una tesis. Persona. 2005;(8):145-70.

Correspondencia: Christian Mejía.

Dirección: Av. Las Palmeras 5713 - Los

Olivos. Lima 39 - Perú.

Teléfono:+51997643516

Correo electrónico: christian.mejia.md@ gmail.com

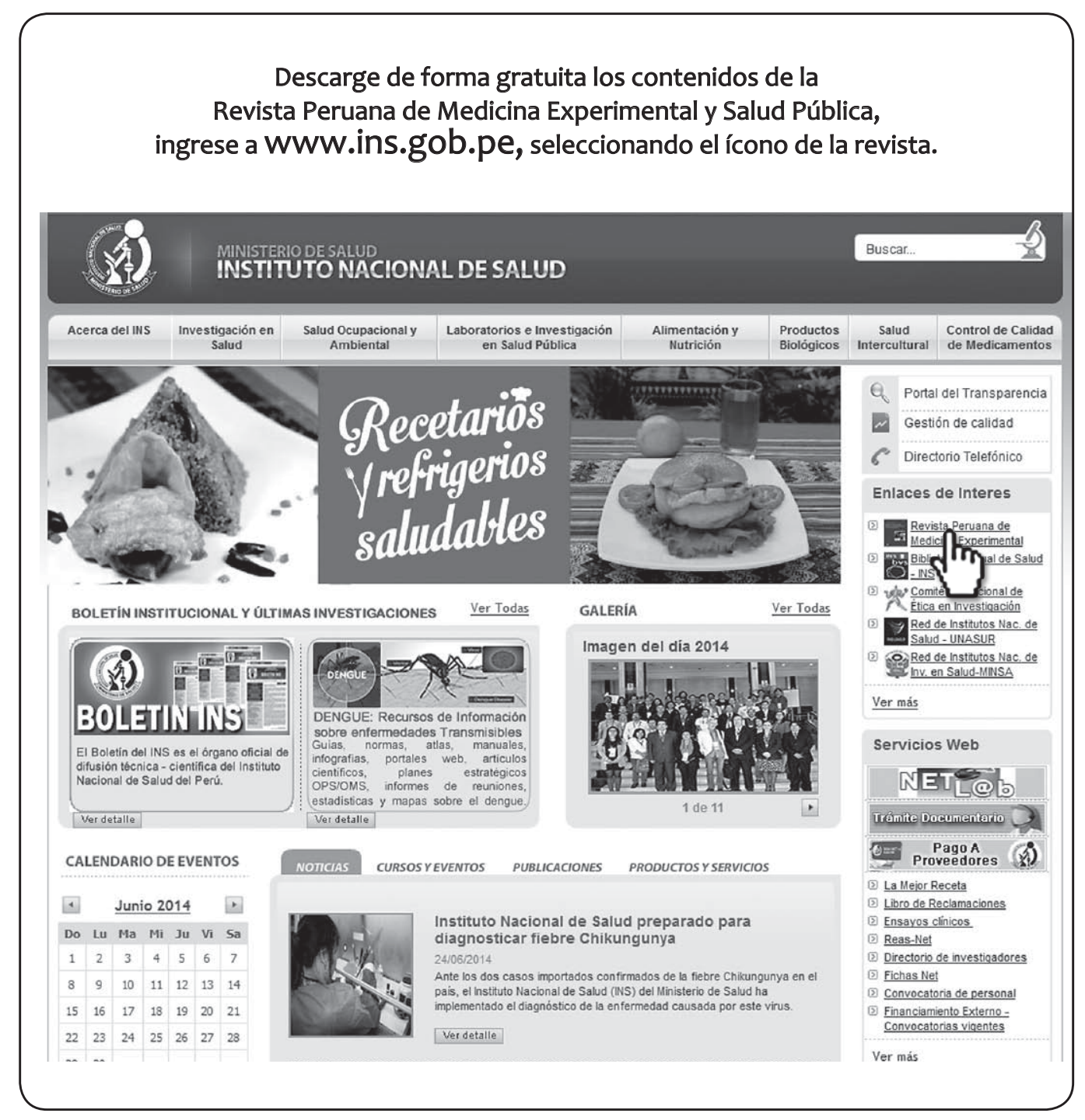

